\title{
Percepción de enfermedad, características de personalidad y dinámica familiar en niños y adolescentes afectados por VIH asistentes a instituciones prestadoras de servicios de salud
}

\author{
Illness perception, personality characteristics and family \\ dynamic in children and teenagers affected by HIV, attended \\ in institutions providing health services
}

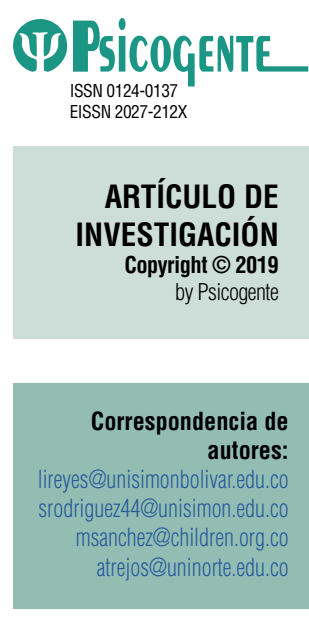

Recibido: 22-01-18 Aceptado: $10-07-18$ Publicado: 01-07-19

\author{
Lizeth Reyes-Ruiz (iD - Sara Rodríguez-Palacio iD \\ Universidad Simón Bolívar, Barranquilla, Colombia \\ Milgen Sánchez-Villegas (iD \\ Children International Colombia, Barranquilla, Colombia \\ Ana María Trejos Herrera (iD \\ Universidad del Norte, Barranquilla, Colombia.
}

Resumen

Objetivo: Describir la percepción de enfermedad, características de personalidad y la dinámica familiar de niños y adolescentes afectados por VIH, teniendo en cuenta sus características sociodemográficas y la revelación o no del diagnóstico.

Método: Es un estudio de tipo cualitativo, con diseño de teoría fundamentada. La muestra estuvo conformada por 14 niños y adolescentes afectados por VIH y diagnosticados desde su nacimiento, entre 6 y 15 años de edad. El muestreo fue no probabilístico intencional o con fines especiales. Se utilizó el test de Machover, el test de la familia y un cuestionario de preguntas abiertas y cerradas sobre percepción de enfermedad.

Resultados: Se encontró que los participantes que no tienen conocimiento sobre su diagnóstico, a diferencia de los que han pasado por un proceso de revelación, poseen una autoimagen perturbada con tendencias a baja autoestima y autoconcepto pobre. A su vez, las conversaciones y los dibujos realizados por ellos sugieren sensación y tendencia a la soledad, inseguridad, deterioro a nivel de las relaciones familiares e interpersonales, evidenciando la existencia de una percepción de enfermedad negativa, que puede incidir en su desarrollo personal y social corroborando lo encontrado por Instone (2000), Trejos, Reyes, Bahamón y Alarcón (2014), Pérez Quiroz, et al. (2013) y Ehrenzweig (2005).

Conclusiones: El presente estudio reconoce la importancia de llevar a cabo procesos adecuados de revelación del diagnóstico para el VIH en niños y adolescentes, permitiendo la mejora de su calidad de vida en pro de su desarrollo biopsicosocial teniendo en cuenta la normalización del ajuste psicológico y mantenimiento del cumplimiento terapéutico, atendiendo a las directrices internacionales sobre el tema de la revelación.

Palabras clave: VIH, revelación diagnóstica, percepción de enfermedad, personalidad, dinámica familiar

Abstract

Objective: This paper aims to describe the perception of illness, personality characteristics and a family dynamic of children and adolescents affected by HIV, considering their social-demographic characteristics and the disclosure or non-disclosure of the diagnosis.

Method: A qualitative study, with a grounded theory design was conducted. For data collection, 14 children

Cómo citar este artículo (APA):

Reyes-Ruiz, L., Rodríguez-Palacio, S., Sánchez-Villegas, M. \& Trejos Herrera, A. M. (2019). Percepción de enfermedad, características de personalidad y dinámica familiar en niños y adolescentes afectados por VIH asistentes a instituciones prestadoras de servicios de salud. Psicogente 22(42), 1-24. https://

doi.org/10.17081/psico.22.42.3491 
and adolescents affected by HIV diagnosed from birth, between 6 and 15 years of age were sampled. This sampling was intentional non-probabilistic or special purposes. The Machover test, the family test and a questionnaire asking open and closed questions to describe illness perception were used.

Results: As a result, it was evident that participants who have no knowledge of their diagnosis, unlike those who have gone through a disclosure process, have a disturbed self-image with tendencies to low self-esteem and poor self-concept. Likewise, conversations and drawings made by them showing tendency to loneliness, insecurity, and deterioration of family and interpersonal relationships, assuming a perception of negative illness, which can affect their personal and social development. Confirming Instone (2000) findings, Trejos, Reyes, Bahamón and Alarcon (2014), Pérez Quiroz, et al. (2013) and Ehrenzweig (2005).

Conclusion: This study recognizes the importance of carrying out adequate diagnostic processes for children and adolescents affected with HIV, allowing the improvement of their quality of life and also, benefiting their psychosocial development, taking into account the normalization of psychological adjustment and preservation of therapeutic adherence. In accordance with international guidelines of HIV disclosure.

Keywords: HIV, diagnostic disclosure, illness perception, personality, family dynamic

\section{INTRODUCCIÓN}

La percepción de enfermedad hace referencia a las representaciones cognitivas que los pacientes construyen con respecto a su enfermedad. Estudios previos en centros de salud de atención primaria han resaltado la importancia de estas creencias sobre la percepción de enfermedad del paciente en relación con la tranquilidad después de pruebas médicas con resultados negativos, la satisfacción después de consultas médicas y la utilización futura de los servicios relacionados con la salud (Weinman et al., 2005) (Frostholm et al., 2005).

En este orden de ideas, la percepción de enfermedad influye en el afrontamiento individual (como la toma de tratamiento) y la respuesta emocional a la enfermedad (Averous, Charbonnier, Lagouanelle-Simeoni, Prosperi, \& Dany, 2018). Estas percepciones son determinantes e importantes en la conducta y se han asociado con una serie de resultados, como la adherencia al tratamiento y la recuperación funcional (Petrie, Jago, \& Devchic, 2007).

Por consiguiente, la comprensión de cómo las personas perciben una enfermedad, tiene implicaciones para la calidad de atención en salud y de cómo interactúan con quienes se ven afectados por alguna enfermedad. Esto es corroborado por Trejos, Reyes, Bahamón, Alarcón y Gaviria (2015), quienes indican que estos procesos de percepción también se evidencian en los familiares y/o cuidadores. En el caso de VIH, por ejemplo, la percepción de la importancia de tomar la medicación por parte del cuidador es clave para preservar la calidad de vida de los niños y asegurar su supervivencia, debido a que lleva a implementar estrategias para motivar al niño y apoyarlo en las dificultades de la ingesta de medicamentos, principalmente relacionadas con el sabor amargo de algunos. A su vez, Croyle y Williams (1991) encontraron 
que las opiniones y juicios hechos sobre una enfermedad, están determinados en parte por las creencias que las personas tienen respecto a estas, pudiendo afectar en gran medida, ya que pueden hacer juicios conscientes o inconscientes en relación con su propia enfermedad influyendo positiva o negativamente en el curso, desarrollo y la posible recuperación.

El virus de la inmunodeficiencia humana $(\mathrm{VIH})$ y las enfermedades de transmisión sexual (ETS) se consideran graves problemas de salud pública debido tanto a los altos costos a los sistemas de salud y bienestar físico, como el malestar emocional de aquellos que sufren de estas enfermedades (Uribe Alvarado, Reyes Ruiz, Trejos Herrera, Bahamón Muñetón, \& Alarcón Vásquez, 2017).

A pesar de la mejoría en las cifras de la aparición de nuevos casos de infección por VIH en Colombia, en el año 2016 ocupó el cuarto lugar entre los países de Latinoamérica con mayor aparición de casos nuevos; esta situación podría estar relacionada con la reemergencia de la infección (UNAIDS, 2017). De acuerdo con las recientes estadísticas, en el país hasta el año 2016, aproximadamente 120.000 niños y adultos viven con VIH. Sin embargo, y teniendo en cuenta el subregistro de casos no diagnosticados, la cifra podría llegar hasta los 150.000 afectados, dentro de los cuales el $0,4 \%$ son niños y adolescentes que se encuentran entre los 0 y 14 años de edad (ONUSIDA, 2016).

Según datos de la Cuenta de Alto Costo (2018), 83.551 registros, se encuentran reportados con corte a 31 de enero de 2017; de esta cifra, 9.396 son nuevos casos. La diferencia de la incidencia entre los periodos de reporte 2015 y 2017 es de 3,7 puntos porcentuales. Esta información se corrobora con otra fuente de investigación en el país: entre 2015 a 2016 la notificación al Instituto Nacional de Salud se incrementó en 15,6\%; adicionalmente, en el informe también se recalca que para el mismo periodo de notificación del año 2016, el dato aumentó en 4,4\% en el año 2017 (Instituto Nacional de Salud, 2017). Por consiguiente, diferentes autores se han interesado por comprender las conductas sexuales de riesgo, debido a que a pesar de existir un adecuado conocimiento de las consecuencias negativas que ello puede acarrear a mediano y largo plazo, su nivel de prevalencia continúa siendo alto. En el caso de los adolescentes, dicha situación se agudiza debido a las condiciones físicas, emocionales y psicológicas de desarrollo y elaboración, que los hacen más vulnerables (Bahamón, Vianchá, \& Tobos, 2014). 
En la sociedad existe un estigma asociado al VIH/SIDA, el cual ha tenido consecuencias muy serias para quienes viven con esta condición. Para muchas personas, vivir con VIH/SIDA implica la necesidad de tener que manejar la discriminación, la marginación y la exclusión (Varas, Serrano, \& Toro, 2004). Igualmente, en concordancia con Nettleton (2002), cualquier enfermedad crónica impacta la vida cotidiana de los afectados tanto en sus relaciones sociales, como en el sentido de sí mismos. Más aún, el diagnóstico de una enfermedad crónica o amenazante constituye el inicio de un largo procedimiento de adaptación, el cual se puede considerar como una crisis (Avelar, Cornejo \& Torres, 2011).

En este sentido, el objetivo de este artículo es describir la percepción de enfermedad, características de personalidad y dinámica familiar de niños y adolescentes afectados por VIH entre 6 y 15 años de edad, asistentes a instituciones prestadoras de servicios de salud. Se parte del supuesto que las reacciones y percepciones frente al VIH/SIDA o cualquier enfermedad varían en cada individuo, dependiendo de factores, tales como: la estructura de la personalidad y mecanismos de adaptación y defensa, la historia previa del individuo, el modo usual de afrontar las situaciones de estrés y crisis, los antecedentes psicopatológicos, la existencia o no de una red de soporte socioemocional y de cómo utilizar estos recursos (Avelar, et al., 2011).

\section{MÉTODO}

\subsection{Diseño}

La presente investigación está enmarcada bajo el enfoque cualitativo de acuerdo con los planteamientos de Hernández, Fernández y Baptista (2010); utiliza la recolección de datos sin medición numérica para descubrir o afinar preguntas de investigación en el proceso de interpretación. El diseño de la investigación se encuentra basado en la teoría fundamentada, su propósito es desarrollar teoría basada en datos empíricos y se aplica a áreas específicas. El diseño de teoría fundamentada utiliza un procedimiento sistemático cualitativo para generar una teoría que explique en un nivel conceptual una acción, una interacción o un área específica.

\subsection{Participantes}

La muestra estuvo conformada por 14 niños y adolescentes, quienes cumplieron los criterios de inclusión: (a) estardiagnosticados con VIH desde su nacimiento,(b) ser asistentes a una institución prestadora de servicios de salud de la ciudad de Barranquilla y (c) tener menos de 18 años de edad. Se 
empleó un muestreo no probabilístico con fines especiales para la elección de los participantes.

\subsection{Instrumentos}

\subsubsection{Test de la figura humana de Karen Machover}

Es una técnica proyectiva de evaluación de la personalidad y rasgos emocionales-sociales, que implica la producción de un dibujo por parte del evaluado, el cual es analizado con base en su contenido o a las características del dibujo (Sánchez de Gallardo \& Pírela de Faría, 2012). Los dibujos productos del test, están íntimamente relacionados con los impulsos, ansiedades, conflictos y compensaciones características de la personalidad de los evaluados (Portuondo, 2007).

\subsubsection{Test de la familia}

Esta prueba permite -a través de la representación gráfica de la familiaobtener elementos de análisis valiosos acerca de las condiciones familiares y de su influencia en el desarrollo afectivo y social infantil encuadrando los aspectos estructurales y funcionales del entorno familiar, así como, de las relaciones que allí se establecen (Lodo-Platone, 2007). Corman (1967), plantea que el test permite explorar la forma como el niño se integra y se relaciona con el sistema familiar, así como las dificultades que experimenta en las interacciones con los miembros de su familia.

\subsubsection{Cuestionario sobre percepción de enfermedad}

Se desarrolló un cuestionario ad hoc sobre percepción de enfermedad constituido por ocho preguntas abiertas, que permiten darle al participante la libertad de responder sin imponerles un límite, las cuales se asocian a su caso particular ayudando a establecer una visión más completa en el contexto del niño. Esto en relación, con lo expuesto por Trejos Herrera, Alarcón Vásquez, Bahamón y Reyes Ruiz (2017), quienes manifiestan que, la percepción de enfermedad en estos menores puede variar desde aquellos que, ante preguntas acerca de la toma de medicamentos $y$, asistencia a la clínica, no evidencian tener percepción subjetiva de su enfermedad hasta los que en sus respuestas muestran una ansiedad ante la experiencia de sentirse enfermos. Las preguntas fueron: ¿Por qué vienes a la IPS?, ¿Qué te han dicho tus padres o cuidadores?, ¿Cómo te dio esa enfermedad y/o síntoma? Para ti, ¿qué es lo peor de estar enfermo?, ¿Tomas algún medicamento?, ¿Para qué son los medicamentos?; Para ti, ¿qué es una enfermedad?, ¿Qué entiendes 
acerca de estar enfermo?, ¿Qué entiendes acerca de estar sano? Finalmente, se indagaron las variables sociodemográficas de los participantes a través de sus cuidadores.

\subsection{Procedimientos}

Se procedió a establecer contacto con las instituciones prestadoras de servicios de salud IPS de la Costa y Vital Salud del Caribe IPS en la ciudad de Barranquilla, mediante carta institucional con visto bueno de la decanatura del programa de Psicología de la Universidad Simón Bolívar para solicitar aval de ingreso a los sujetos de estudio. Como documentos anexos a la carta institucional fueron enviados el protocolo de la investigación, el formulario de consentimiento informado y copia del instrumento de medición para someterlos al respectivo estudio de los Comités de Ética de ambas entidades. Posteriormente, fueron concretadas reuniones con las personas encargadas de este tipo de solicitudes en ambas entidades para explicar en forma verbal el Protocolo de la Investigación. Obtenido el aval de ingreso a estas instituciones, se asignaron las fechas en que se procedería con el proceso de recolección de datos con los niños, adolescentes y sus cuidadores con el respectivo acompañamiento del funcionario(a) de la entidad. Las entrevistas con cada evaluado tuvieron una duración de $\mathbf{4 0}$ minutos aproximadamente, en los que se aplicaron los instrumentos mencionados con anterioridad.

\subsection{Análisis de datos}

Después de la aplicación de los instrumentos proyectivos se hizo la interpretación de todos los dibujos resultantes; se contó con asesorías de expertos en aplicación, manejo e interpretación de las pruebas proyectivas.

Para la sistematización de las características de personalidad, fue necesario dividirlas en tres categorías importantes (El Ello-ID, Yo-Ego, y Súper Yo-Súper Ego). Se identificaron los signos patognomónicos encontrados en cada caso, y luego se procedió al análisis en conjunto identificando los rasgos de personalidad en común.

La sistematización de la dinámica familiar se interpretó teniendo en cuenta tres planos: El gráfico, de estructuras formales y de contenido, analizando las características en común que se encontraron en cada evaluado con respecto a su dinámica familiar. 
Para la sistematización del cuestionario de percepción de enfermedad y variables sociodemográficas, se analizaron los datos en el paquete estadístico para las Ciencias Sociales SPSS versión 22.

\subsection{Conflicto de interés}

Los autores declaran que no tienen ningún conflicto de interés potencial con respecto a la investigación, autoría y/o publicación de este artículo.

\section{RESULTADOS}

\subsection{Variables Sociodemográficas}

Tabla 1.

Variables sociodemográficas de niños y adolescentes afectados con VIH.

\begin{tabular}{ccc}
\hline VARIABLE & ÍTEM & FRECUENCIA \% \\
\hline \multirow{2}{*}{ Edad } & Entre 6 y 8 años & $8(58 \%)$ \\
& Entre 9 y 11 años & $2(14 \%)$ \\
Entre 12 y 14 años & $2(14 \%)$ \\
Entre 15 y 17 años & $2(14 \%)$ \\
& Femenino & $5(36 \%)$ \\
Escolaridad & Masculino & $9(64 \%)$ \\
& Pre-escolar & $1(7 \%)$ \\
Conocimiento del diagnóstico & Primaria & $9(65 \%)$ \\
Diagnóstico & Secundaria & $3(21 \%)$ \\
VIH cuidador & No estudia & $1(7 \%)$ \\
Diagnóstico madre VIH & Sí & $4(29 \%)$ \\
& No & $10(71 \%)$ \\
Diagnóstico Padre VIH & Sí & $4(29 \%)$ \\
& No & $10(71 \%)$ \\
Diagnóstico & Sí & $14(100 \%)$ \\
Hermanos VIH & No & $0(0 \%)$ \\
& Sí & $4(29 \%)$ \\
Estrato & No & $10(71 \%)$ \\
& Sí & $1(7 \%)$ \\
Régimen de salud & No & $13(93 \%)$ \\
& 1 & $11(79 \%)$ \\
& 2 a 3 SMLV & $2(14 \%)$ \\
& Contributivo & $1(7 \%)$ \\
& 3 Subsidiado & $7(50 \%)$ \\
& & $6(43 \%)$ \\
& Menor a 1 SMLV & $1(7 \%)$ \\
& $2(14 \%)$ \\
& & $12(86 \%)$ \\
\hline & &
\end{tabular}

Producto de la revisión de los resultados obtenidos sobre las variables sociodemográficas de los participantes afectados por VIH se encontró que, el rango de edades osciló entre 6 y 15 años. Referido al género, son en su mayoría pertenecientes al género masculino. En cuanto al conocimiento del 
diagnóstico por parte del menor se encuentra que el $71 \%$ de los menores consultados no conocen su diagnóstico y el $36 \%$ restante tiene conocimiento de este.

Del nivel de escolaridad de los participantes, se encontró que el $65 \%$ cursaba primaria, el $21 \%$ secundaria, el $7 \%$ pre-escolar y el $7 \%$ restante no estudiaba. En lo referente al diagnóstico para VIH del cuidador, 4\% de ellostenían un diagnóstico positivo para VIH. En concordancia los resultados obtenidos, se encontró que el $100 \%$ de las madres de los participantes tienen un diagnóstico para $\mathrm{VIH}$, sin embargo, solo el $29 \%$ de los padres están afectados, mientras que el $71 \%$ restante no tienen este diagnóstico; el 7\% de los hermanos(as) están afectados con VIH y el 93\% restante no. Relativo al estrato socioeconómico del hogar, el $79 \%$ de los hogares se encuentran en el estrato socioeconómico 1, un $14 \%$ en estrato socioeconómico 2 y el $7 \%$ restante en estrato socioeconómico 3. En relación a los ingresos económicos del hogar de los participantes, los resultados arrojaron que el $50 \%$ de hogares cuentan con ingresos menores a $1 \mathrm{SMLV}$, un $43 \%$ de hogares que tienen ingresos de 1 SMLV, mientras que solo el $7 \%$ cuenta con ingresos de 2 a 3 SMLV. Respecto al régimen de salud del menor la mayoría pertenece al régimen subsidiado con un porcentaje del $86 \%$ de la población del estudio, mientras que solo el $14 \%$ pertenece al régimen contributivo.

\subsection{Percepción de enfermedad}

\begin{tabular}{ccc}
\hline PREGUNTA ABIERTA & ÍTEM & FRECUENCIA (\%) \\
\hline ¿Por qué vienes a la IPS? & Control del estado de salud o para evitar una & $6(42.85)$ \\
& enfermedad & $3(21.42)$ \\
& Ingesta de medicamentos & $3(21.42)$ \\
¿Cómo te dio esa enfermedad? & Control sobre curso del VIH & $1(7.14)$ \\
& Desconocen el motivo & $1(7.14)$ \\
Para ti, ¿̇qué es lo peor de estar & Engaño con otra enfermedad & $7(50)$ \\
enfermo? & No sabe & $4(28.57)$ \\
& Madre causante de la enfermedad & $2(14.28)$ \\
& Producto de la gripa & $1(7.14)$ \\
& Deterioro de salud & $6(42.85)$ \\
& NTomas algún medicamento? & $4(28.57)$ \\
& Sí, pero no conoce el nombre de los medicamentos. & $2(14.28)$ \\
& Sí, conoce los horarios del medicamento. & $1(7.14)$ \\
& No sabe/No responde. & $1(7.14)$
\end{tabular}




\begin{tabular}{|c|c|c|}
\hline PREGUNTA ABIERTA & ÍTEM & FRECUENCIA (\%) \\
\hline ¿Para qué son los medicamentos? & $\begin{array}{c}\text { No responde } \\
\text { Presencia de un virus (no saben cuál) } \\
\text { Prevenir una enfermedad y estar saludables. } \\
\text { No sabe } \\
\text { Por el VIH }\end{array}$ & $\begin{array}{l}4(28.57) \\
4(28.57) \\
3(21.42) \\
2(14.28) \\
1(7.14)\end{array}$ \\
\hline Para ti, ¿qué es una enfermedad? & $\begin{array}{c}\text { Tener las defensas bajas } \\
\text { Asociación con médicos, hospitales } \\
\text { No responde } \\
\text { Fiebre/Gripa } \\
\text { No poder salir } \\
\text { Estar contagiado de un virus }\end{array}$ & $\begin{array}{l}4(28.57) \\
3(21.42) \\
3(21.42) \\
2(14.28) \\
1(7.14) \\
1(7.14)\end{array}$ \\
\hline $\begin{array}{c}\text { ¿Qué entiendes acerca de estar } \\
\text { enfermo? }\end{array}$ & $\begin{array}{c}\text { No responde } \\
\text { Limitaciones para hacer } \\
\text { actividades diarias } \\
\text { Gripa/Fiebre } \\
\text { Ingesta de medicamentos } \\
\text { Miedoso } \\
\text { Algo psicológico }\end{array}$ & $\begin{array}{l}5(35.71) \\
3(21.42) \\
3(21.42) \\
1(7.14) \\
1(7.14) \\
1(7.14)\end{array}$ \\
\hline $\begin{array}{c}\text { ¿Qué entiendes acerca de estar } \\
\text { sano? }\end{array}$ & $\begin{array}{l}\text { Sanos, vivos, fuertes y saludables } \\
\text { No responde } \\
\text { Jugar y hacer actividades cotidianas } \\
\text { Buen comer } \\
\text { Tomar los medicamentos }\end{array}$ & $\begin{array}{l}6(42.85) \\
4(28.57) \\
2(14.28) \\
1(7.14) \\
1(7.14)\end{array}$ \\
\hline
\end{tabular}

Al revisar las respuestas obtenidas por los participantes en lo referente con la percepción de enfermedad se evidenció y se corroboró desde una perspectiva psicoanalítica y pensamiento freudiano, la existencia de un Principio de Realidad en donde se exhiben mecanismos de defensa, los cuales el Yo activa para evitar la angustia y permitir la funcionalidad del individuo. identificándose la resistencia, represión, negación y rechazo frente a una idea o situación relacionada con el conocimiento de la enfermedad al no responder las preguntas referentes con su percepción. La mayoría de los participantes no tenía conocimiento de su diagnóstico para VIH, por lo que las explicaciones recibidas de su parte sobre el porqué asistían a la institución prestadora de servicios de salud, la ingesta de medicamentos y los exámenes médicos de control a los que son expuestos, estaban referidas directamente a las explicaciones que sus cuidadores les daban relacionadas con otros diagnósticos leves, como gripas, fiebres, tos y dolores de garganta.

\subsection{Personalidad}

Teniendo en cuenta los resultados obtenidos en la aplicación de los instrumentos, su interpretación y análisis, será preciso mostrar que existen diferencias significativas referentes a la evaluación de la personalidad de los participantes, considerando que algunos de ellos habían pasado por un proceso de revelación de diagnóstico adecuado, otros tenían conocimiento de 
una manera negativa y la gran mayoría desconocía que tenía un diagnóstico positivo para VIH.

Para tener una mayor comprensión de los resultados obtenidos de la evaluación de la personalidad de los participantes se tomó como referente explicativo el enfoque psicodinámico, quien concibe la personalidad como un proceso dinámico y cambiante, en donde entran en juego la relación e interacción de las diferentes estructuras psíquicas del individuo (Ello o Id, Yo o Ego y Súper Yo o Súper Ego), las cuales de manera separada poseen unas características y funciones específicas que al integrarse y relacionarse dan como resultado diferentes rasgos característicos y un esbozo global de la personalidad de los niños, niñas y adolescentes evaluados.

Con relación a las características de personalidad de todos los sujetos evaluados se encontró en la categoría Ello o ld el signo patognomónico común de la línea gruesa y presión fuerte, sinónima de agresividad, impulsividad y rebeldía. A su vez, en la subcategoría inconsciente, los signos que se evidenciaron fueron brazos extendidos, inclusiones de órganos anatómicos (corazones), interpretándose como represión e incomodidad, lo cual se corroboró con la observación al realizar las respectivas preguntas de apoyo, se encontró que en todos los sujetos se hizo evidente la presencia de demandas de afecto, esto mediado en gran medida por su dinámica familiar.

En cuanto a las características de personalidad en la categoría del Yo y/o Ego, se pudo evidenciar que todos los participantes se encontraron situados en tiempo y espacio, indicador de la subcategoría de principio de realidad. Por otra parte, se observó en la subcategoría juicio, cuyo signo patognomónico es el énfasis en la cabeza, interpretado como un índice de preocupación y percepciones hacia un tema en específico. Es por esto que se esboza en los participantes un proceso de percepción sobre su enfermedad, acarreando notables diferencias según el conocimiento de su diagnóstico, ya que, quienes han pasado por un proceso de revelación de diagnóstico adecuado tienen un manejo apropiado sobre la temática, terminología asociada y correcto uso de tecnicismos; sin embargo, quienes conocen su diagnóstico de una manera inadecuada perciben su enfermedad como algo negativo, que los lleva a tener una asociación del proceso de enfermar con la muerte, lo que causa gran angustia, sentimientos de depresión, aislamiento y deterioro en su formación personal. Además, no poseen un manejo adecuado sobre lo que implica tener un diagnóstico positivo para VIH. Simultáneamente, los participantes que desconocen su diagnóstico elaboran procesos sobre la percepción 
de enfermedad más primitivos puesto que el desconocimiento los lleva a tener un sinnúmero de posibilidades tanto positivas como negativas sobre lo que implica estar "enfermo" según lo que es escuchado en el medio, lo comentado en los espacios donde se mueven, lo dicho por personas cercanas a su círculo familiar y social y los comportamientos discriminatorios en tareas habituales de los demás frente a ellos.

En la subcategoría sentido de la realidad del mundo y de sí mismo, en los que los signos patognomónicos son dibujos en la parte baja, cuadrante inferior izquierdo, la inclusión de cuerpos de agua (ríos, cascadas, Iluvia, etc.), figuras pequeñas, son asociadas con un Yo disminuido, autoestima baja y autoconcepto pobre; pies pequeños, reforzados, puntiagudos y figura mal equilibrada y/o mal parada, línea confusa y fragmentada, se puede interpretar como un índice de inseguridad, baja autoestima, timidez, sentimientos de soledad, depresión, inseguridad y Yo debilitado. Estos rasgos fueron encontrados en los participantes que desconocen su diagnóstico y quienes lo conocen producto de una mala práctica. En contraste y constituyéndose como minoría algunos evaluados cuentan con un autoconcepto adecuado, buena autoestima, energía vital, sentimientos de fortaleza y estabilidad, producto al conocimiento de su diagnóstico, gracias a un buen proceso de revelación y el acompañamiento de personas expertas en la materia.

En la subcategoría de regulación y control de instintos, afectos e impulsos, siendo la dependencia oral su signo patognomónico, hallándose en la boca cóncava u oralmente receptiva, línea de botones asociada con dependencia materna y dependientes, manos y dedos dibujados en forma de lanzas, picos u objetos puntiagudos que indican agresividad manifiesta, se encontró en la gran mayoría la dependencia oral, tendencia a la agresividad y que en algunos existe dependencia materna y emocional, viéndose asociado a las fuertes relaciones de protección, lazos de afecto y cuidado por parte de los cuidadores y/o padres, quienes producto del estigma social frente al $\mathrm{VIH}$, buscan evitar cualquier angustia, trauma o situación negativa a los participantes. A pesar de saber o no sobre su diagnóstico los participantes muestran rasgos marcados de agresividad y de cuidado o precaución frente al medio que les rodea, esto producto de situaciones de la vida cotidiana que en apariencia pueden pasar desapercibidas, pero se constituyen como hostiles y negativas al estar relacionadas con su diagnóstico, producto del estigma y desconocimiento social sobre la enfermedad. 
También se evidenció en la subcategoría relaciones objétales, donde su signo patognomónico es deterioro de las relaciones interpersonales, la omisión de algún rasgo facial, manos sombreadas vigorosamente, las cuales sugieren culpabilidad con relación a impulsos agresivos. Dirección y fluencia de las líneas de los brazos relacionadas con el grado y la espontaneidad de contacto interpersonal dentro del medioambiente, lo cual en la gran mayoría de los evaluados se manifestó como una tendencia al aislamiento, deterioro a nivel de las relaciones interpersonales, retraimiento, ambivalencia materna. Sin embargo, algunos de los participantes muestran buenas relaciones personales y adecuación al medio, producto de los procesos de revelación diagnóstica individuales positivos.

Por otra parte, en la subcategoría procesos del pensamiento, donde el signo patognomónico es la ubicación en el plano superior izquierdo, indicando amplia imaginación característica en niños, pelo en forma desordenado e irregular indicador de ideas fijas y recurrentes. Plano central indicando movimiento progresivo y evolutivo adecuado. Dificultades a nivel de lenguaje, observación conductual, dibujos muy pobres, sin terminar, sin esquematizar, posibilidad de daño a nivel neuropsicológico, se encontró que la gran mayoría de los sujetos están situados en un plano imaginativo normal para su edad, también presentan ideas fijas y recurrentes, las cuales tienen una relación directa en el proceso de enfermar, algunos se encontraron en un plano imaginativo inadecuado para su edad, aunque están situados en un plano central para su edad. Resalta el caso de uno de ellos quien presenta dificultades a nivel de lenguaje y posible dificultad a nivel neuropsicológico.

Se evidenció que en la subcategoría de regresión adaptativa al servicio del yo, cuyo signo patognomónico es ubicación del dibujo en el lado izquierdo, muestra un retraimiento o dirección hacia el pasado, la gran mayoría de los niños presentaron rasgos regresivos normales para su edad.

En la subcategoría de funcionamiento defensivo se evidencian signos patognomónicos tales como la omisión de las orejas muestra relación a la crítica u opinión social. Se encontró que todos los sujetos presentaron represión y resistencia notable, negación y sensibilidad hacia lo que pueda escuchar, indicando que son sensibles a la opinión y crítica y que tienen una hipervigilancia ante lo que pueda decirse en su medio sobre su estado de salud. 
Se encontró que la gran mayoría tuvo una adecuada barrera a los estímulos y que todos los sujetos tienen un adecuado funcionamiento autónomo y funcionamiento sintético-integrativo.

En la subcategoría dominio de competencia, donde los signos patognomónicos son contornos imprecisos (manos) u oscurecidos, índice de falta de confianza en los contactos sociales, en la propia productividad o ambas; brazos dibujados frágiles o consumidos, que indican deficiencia y debilidad, ya sea como realidad física o reacción psíquica; piernas dibujadas débiles, delgadas, desproporcionadas, temblorosas o pequeñas, asociadas con sentimientos de debilidad y decadencia, se pudo comprobar que la gran mayoría de los niños presentó sentimiento de decadencia y debilidad, sin embargo, algunos tuvieron sentimientos orientados al cuidado, protección y seguridad propios.

En la categoría Superyó-Superego, donde una de las subcategorías es la conciencia moral y auto-observación cuyos signos patognomónicos son cuellos altos y delgados índice de moralistas, educados, rígidos y la identificación sexual en el primer dibujo realizado, se pudo observar la identificación adecuada con su propio sexo. Se debe agregar que algunos presentaron un Súper Yo dominante, comportamiento moralista, rígido y educado.

Por otro lado, en la subcategoría formación de ideales, la cual es evidenciada en la historia vital, el discurso y en las preguntas de apoyo respondidas por los participantes, se evidenció que la gran mayoría de los sujetos presenta una limitación con el medio a pesar de tener una proyección hacia el futuro. Algunos muestran poca adaptación social o no se evidencia. Para finalizar, en la subcategoría introyección de normas y reglas se pudo constatar que la gran mayoría de los niños tiene una adecuada introyección de normas y solo uno manifestó rebeldía.

\subsection{Dinámica familiar}

A continuación, se presentarán los resultados hallados a través del Test de la familia, cuyo objetivo es el análisis de las relaciones interpersonales, relación del niño con cada uno de los miembros de su familia teniendo en cuenta los planos que evalúa el test, gráfico de estructuras formales y de contenido con sus respectivas subcategorías y signos patognomónicos, los cuales indican y guían la evaluación, siempre tomando en cuenta la etapa del desarrollo cronológico en la que este se encuentra, así como la fase del desarrollo emocional. 
Dentro de la categoría plano gráfico, cuyo signo patognomónico es manifestado por el grosor, intensidad del color o las marcas dejadas en el papel, se hizo evidente la subcategoría de fuerza en el trazo en los dibujos realizados.

En la subcategoría amplitud con signos patognomónicos de movimiento amplio como la ocupación de buena parte de la hoja, movimientos restringidos, líneas curvas o largas con pequeños trazos, se encontró que todos los evaluados presentan inhibición de expansión vital indicando una presencia débil de su desarrollo en su núcleo familiar. De igual modo se evidenciaron en las subcategorías ritmo del trazo y sector de la página con signos patognomónicos de ritmo estereotipado: el mismo estilo en todas las figuras, en fila; ritmo desordenado: cada personaje de otro estilo, que la mayoría de los evaluados se encuentran en la expansión imaginativa y pérdida de espontaneidad y apego a las reglas.

De igual manera, en la subcategoría sector de la página con signos patognomónicos tales como ubicación en el sector inferior, sector superior, dibujo de la mitad a la izquierda, sector derecho, sector en blanco, dibujo hecho de derecha a izquierda, dibujo hecho de izquierda a derecha, se pudo evidenciar que en algunos participantes se encuentran sentimientos de depresión y apatía y sentimientos de inferioridad.

En la categoría de estructuras formales, la subcategoría sensorial-racional y mixto, donde sus signos patognomónicos son sensorial: uso de líneas curvas (dinamismo); racional: uso de líneas rectas y los ángulos predominan sobre las curvas; mixto: líneas curvas y rectas, se encontró que en la mayoría existe una estructura de tipo sensorial, pero tres de ellos hay en estructura racional y algunos evidencian una estructura mixta.

Simultáneamente a las características anteriores, dentro de la categoría contenido, la subcategoría angustia frente a un peligro exterior, se pudo ver manifiesta en la mayoría de los evaluados, de los cuales cinco de ellos realizan una inversión de papeles; y solo en uno se evidencia regresión. Por otra parte; se encontró que en cinco de los evaluados no se observa la subcategoría angustia frente a un peligro interior, otros cinco evidencian sentimientos de agresión; y una pequeña parte, evidencian autodesvalorización. De la misma manera, se observó en la gran mayoría de los evaluados en la subcategoría preferencias e identificaciones, una identificación real, dentro de las cuales, cuatro de los sujetos evidencian deseos de una familia ideal. 
De igual modo, se identificó en la mayoría de los evaluados una desvalorización, donde seis de ellos evidencian la característica anterior paralela a una relación distante de sus padres, mientras que en cinco de los evaluados se observa una valorización como defensa contra la angustia.

\section{DISCUSIÓN}

Los resultados obtenidos en esta investigación muestran y corroboran lo encontrado por diversos autores frente al VIH/SIDA, la notable existencia, aún, de muchos interrogantes respecto a la etiología de la enfermedad, lo que puede generar una distorsión en la imagen que las personas tienen respecto a esta y en consecuente de sí mismas, debido a que la carga social y el grupo con el que se ha identificado la enfermedad, ha sido caracterizado histórica y socialmente desde lo "negativo", lo que lleva a evitar hablar del tema por temores al rechazo, la exposición y la discriminación (Abadía-Barrero \& Larusso, 2006; Boon-Yasidhi, et al., 2005; Lester, et al., 2002; Kouyoumdjian, Meyers, \& Mtshizana, 2005; Ledlie, 1999; Lipson, 1993; Oberdorfer, et al., 2006; Tasker, 1992; Waugh, 2003).

Hay que mencionar, además que los pacientes pediátricos con el virus de inmunodeficiencia humana (VIH) y síndrome de inmunodeficiencia adquirida (SIDA) ahora viven más tiempo con la mejora de su calidad de vida, lo cual hace que la revelación sea un proceso esencial (Committee on Pediatric AIDS, 1999; Lee \& Johann-Liang, 1999).

Siguiendo este orden de ideas, en coherencia con los hallazgos de Instone (2000) y teniendo en cuenta los postulados de Bellack y Fleming (1996), se encontró que al evaluar a los participantes, estos tienen características de personalidad únicas, afectados con VIH en virtud de su edad cronológica y capacidades de desarrollo de percibir y entender las enfermedades que amenazan la vida, son potencialmente conscientes de la naturaleza de su enfermedad, incluso si nunca habían sido informados. Pues en el Yo, se ordenan las dimensiones físicas, cognitivas, afectivas y sociales, a partir de la satisfacción, la frustración y la realidad interna y externa, emergen los mecanismos de defensa, se produce la adaptación y estructuración psíquica (Bustos \& Russo, 2018). Por estas razones, algunos de los niños sospechaban la verdad de su enfermedad, pero pretendían no saberlo. Al igual, se encontró que, los participantes argumentan ir al médico u hospital para que les realicen controles de salud, o exámenes de sangre, los cuales se pueden relacionar con las respuestas proporcionadas por los participantes del porqué asisten a la IPS. Algunas de las razones para retrasar la entrega del diagnóstico que 
reportaran profesionales de salud y cuidadores de los menores afectados se relacionan con evitar daño psicológico o estrés emocional al menor, temor a causar situaciones de estigmatización ante la revelación involuntaria a otros, y falta de capacitación respecto al procedimiento y edad para entregar esta información (Trejos, Mosquera, \& Tuesca, 2009; Abadía-Barrero \& Larusso, 2006; Lester, et al., 2002; Oberdorfer, et al., 2006; Kouyoumdjian, Meyers, \& Mtshizana, 2005; Boon-Yasidhi, et al., 2005). Se corrobora entonces, que las personas tienen una concepción de salud y enfermedad que influye en cómo reaccionan a los síntomas percibidos. Las llamadas percepciones (o esquemas) de la enfermedad son las representaciones organizadas de la enfermedad que se adquieren a través de experiencias de determinadas patologías que haya padecido el mismo sujeto o sus familiares o amigos (Taylor, 2007).

Todavía cabe señalar que la presencia de una enfermedad crónica transmisible como el VIH/SIDA en un individuo puede redundar en el ámbito familiar, sobre todo cuando se presenta en jóvenes, los cuales están en una etapa de transformación y aceptación en una sociedad, personalización de género, cambios conductuales en el interior de la familia (Trejos Herrera, Bahamón, \& Gaviria García, 2017). Al mismo tiempo, Cummings y Davies (2002) afirman que cuando un adolescente es expuesto a ambientes familiares incongruentes o disfuncionales es más probable que desarrolle desajustes emocionales o cognitivos. Así, por ejemplo, la carencia de redes de apoyo en el ambiente familiar en los adolescentes afecta su vinculación psicosocial, el pobre funcionamiento familiar, la presencia de crisis familiares, la disfuncionalidad, la poca expresividad, la mala comunicación y la desorganización familiar.

En este sentido, se pone en evidencia una de las problemáticas y discusiones que han surgido desde el estudio del VIH/SIDA, la revelación del diagnóstico a menores de edad, ya que, representa un gran desafío para los padres, cuidadores y profesionales de la salud, debido al estigma asociado con esta enfermedad, a sus modos de transmisión y el secreto que rodea a menudo el diagnóstico (Nehring, Lashley, \& Malm, 2000; Instituto Colombiano de Bienestar Familiar, Save the Children, Unicef, \& Universidad del Norte, 2006). Esta situación hace frente a la visión y concepción que se tiene sobre el VIH, como una enfermedad de gran carga social, que genera frecuentemente comportamientos de rechazo, distanciamiento, señalamiento hacia quienes tienen el diagnóstico, e incluso entre las mismas personas que viven con la infección (Guevara-Sotelo \& Hoyos-Hernández, 2018) afectando así, el autoconcepto familiar, el cual se entiende como la percepción que tiene el 
sujeto de su implicación, participación e integración en el medio familiar. El significado de este factor se relaciona con el afecto y la confianza establecida en la relación con los padres, así como con la percepción de felicidad y apoyo que se percibe en la familia y el hogar (Montoya, Pinilla, \& Dussán, 2018). De ahí que Varela, Salazar y Correa (2008) refieren en su estudio que para un mejor abordaje se debe distinguir al paciente como ser humano que asume su propia percepción de la realidad, y tiene una forma individual de actuar frente a ella; por lo tanto, se tendrá en cuenta su estado emocional, valores, cultura, tradiciones, creencias, las cuales pueden ser aspectos que influyan en los proyectos futuros del individuo.

Por consiguiente, los retos para el abordaje del VIH entre las ciencias de la salud y las ciencias sociales son grandes. Es claro que el VIH hace necesario el abordaje integral, en donde no solo se trabaje el paciente, sino también en el acompañamiento a las familias, la red de apoyo de estas personas e incluso en personas que no tengan la enfermedad, todo lo relacionado con el estigma social, la discriminación y el estigma sentido. De esta manera, se reconoce la necesidad de hacer un abordaje biopsicosocial respecto a esta temática en particular (Guevara-Sotelo \& Hoyos-Hernández, 2018). Así mismo, una explicación oportuna, precisa y llevada a cabo con empatía, dentro de un proceso seguro y planificado de revelación es preferible que dejar expuesto al niño(a) a revelaciones accidentales que pueden ir en detrimento de su ajuste psicológico y adherencia terapéutica (Trejos Herrera, Alarcón Vásquez, Reyes Ruíz, \& Bahamón, 2017).

Dicho lo anterior, el desafío que los fenómenos humanos han representado para los intereses investigativos, puede reflejarse en la realización de estudios que conciben al sujeto no solo desde el sentido de un desarrollo único, sino además como el producto de una historia familiar; esto se genera, gracias a la motivación en propuestas comprensivas sobre lo subjetivo y su modo de articulación cultural (Mass, 2013).

\section{CONCLUSIONES Y RECOMENDACIONES}

En consonancia con la revisión teórica y con los resultados encontrados, se puede evidenciar la gran importancia que las características de personalidad y las relaciones intrafamiliares tienen en la formación de la percepción de enfermedad en los participantes de este proyecto.

A manera de conclusión, se encontró que en la mayoría de los casos, los participantes mostraron gran resistencia al hablar respecto a la enfermedad 
- desconocida- en muchos casos, manifestándose como una evitación de la realidad mostrando la inminente posibilidad a resistir y aceptar lo que ellos ya saben y perciben sobre su enfermedad, dado que gran parte de los participantes conocen el nombre de los medicamentos, convirtiéndose esto en un factor de riesgo puesto que están expuestos a conocer su diagnóstico de una manera inadecuada. En este sentido, cuando el niño llega a conocer su diagnóstico en condiciones inapropiadas, puede alterar su estado psicológico, el ajuste, la adherencia al tratamiento, el funcionamiento de la familia y el proceso de adaptación a vivir con el diagnóstico (Trejos A. M., et al., 2014).

Siguiendo este orden de ideas, al tener una capacidad cognitiva para asociar el proceso de enfermedad con la ingesta diaria de medicamentos, los participantes generan pensamientos y desarrollan una percepción sobre la enfermedad. En tal sentido, los participantes que desconocen su diagnóstico sospechan y generan percepciones negativas que influyen en su personalidad y en su forma de ver la vida asociando así la enfermedad con no poder desempeñar actividades normales e importantes como jugar, lo cual les genera -como planteaba Instone (2000)-, angustia emocional, sentimientos de soledad y una autoestima pobre, generando un desajuste psicológico que no les permite ser adherentes al tratamiento. Caso contrario a los participantes que conocen su diagnóstico gracias a un buen proceso de revelación.

Cabe mencionar además que, emerge el papel fundante de la familia en el desarrollo del joven y su función socializadora que hace más vulnerable al sujeto hacia determinadas formas de percibirse a sí mismo y al mundo. Interpretaciones que pueden impulsar visiones positivas o negativas sobre las capacidades del joven para afrontar las demandas del medio, sentidos y expectativas hacia el presente y el futuro (Kwok \& Shek, 2009).Por otra parte, se puede evidenciar lo encontrado por Pérez Quiroz, et al., (2013), que los problemas de comunicación, tanto con la madre como con el padre, pueden facilitar la aparición de síntomas depresivos en los hijos, esto porque los padres de familia y/o cuidadores siguen proporcionando respuestas que no son reales y claras para evitar un sufrimiento en los participantes. Es de vital importancia que se cambie esta práctica, ya que está comprobado que revelar el diagnóstico a los niños de una manera adecuada no genera un desajuste psicológico y puede ayudar a que se establezcan mejores canales de comunicación entre los familiares. 
También es importante resaltar que los padres, al tratar de dar respuestas persuasivas y engañosas, generan desconfianza y se pierde credibilidad en las figuras cuidadoras o padres de familia, ya que los niños sí logran percibir que están en condiciones diferentes a sus pares (Tasker, 1992). Es fundamental entonces, que el momento de revelación de diagnóstico sea un proceso sumamente positivo, para que los niños tengan una percepción de enfermedad que les permita desarrollarse funcionalmente en la sociedad y prolongar su adherencia al tratamiento y mejorar su calidad de vida.

En síntesis, con los resultados encontrados, es necesario realizar un proceso psicoeducativo con los padres de familia y/o cuidadores, para que puedan ofrecer mejores respuestas a las curiosidades de los niños sobre su enfermedad; también que estén preparados y contribuyan a ser buenos canales de comunicación entre los familiares. Es posible afirmar que la familia, y principalmente los padres, son importantes en el desarrollo del adolescente, ya que dependiendo del estilo parental empleado pueden ser considerados como factor protector para evitar que los jóvenes se involucren en diversas conductas de riesgo, entre ellas el suicidio (Pérez Quiroz, et al., 2013).

Así mismo, se plantea capacitar a la comunidad, con el objetivo de reducir la estigmatización y la vulneración de los derechos de las personas con el diagnóstico de afectación para VIH/SIDA, producto del desconocimiento que aún se tiene respecto a la enfermedad. Es importante continuar con la labor informativa y educativa para que se conozca y comprenda mejor la infección, las verdaderas vías de transmisión y no las procedentes del imaginario colectivo, las conductas de riesgo y las medidas de bioseguridad necesarias; en resumen, todo aquello que pueda ayudar a disminuir ciertas reacciones, actitudes y prevenciones ante las personas (Trejos, et al., 2009).

Agradecimiento: A los equipos interdisciplinarios que participaron: IPS de la Costa y Vital Salud del Caribe IPS por la confianza depositada en nuestro equipo y por ponernos en contacto con su más importante razón de ser: los pacientes y sus familias; a ellos les debemos nuestro más profundo agradecimiento. Es bien conocido el impacto del VIH/SIDA en las familias, entre las cuales es la pérdida de apoyo social y familiar, el estigma y la discriminación, y el impacto físico, psicológico y económico. A pesar de esto, las familias que participaron en esta investigación enfrentaron con valor y fuerza sus temores asociados a la revelación del diagnóstico de VIH a sus niños y jóvenes. 
Financiamiento: Proyecto de Investigación Conductas y Prácticas de Riesgo de Jóvenes en Contextos Académicos. Acto administrativo: AP 03040020713 de la Universidad Simón Bolívar. Barranquilla-Colombia.

\section{REFERENCIAS}

Abadía-Barrero, C., \& Larusso, M. (2006). The disclosure model versus a developmental illness experience model for children and adolescents living with HIV/ AIDS in Sao Paulo, Brazil. AIDS Patient Care and STDs, 20(1), 36-43. https://doi. org/10.1089/apc.2006.20.36

Cuenta de Alto Costo (2018). Situación del VIH SIDA en Colombia 2017. Bogotá: Colombia: Fondo Colombiano de Enfermedades de Alto Costo - Cuenta de Alto Costo [CAC]; Disponible: https://cuentadealtocosto.org/site/images/Publicaciones/2018/Situacion_VIH_2017..pdf

Avelar Rodríguez, V. Y., Cornejo Gómez, I. B. \& Torres Ayala, J. D. (2011). Efectos psicológicos en personas de ambos sexos entre las edades de 20 a 50 años diagnosticadas con el VIH en el periodo de enero de 2006 a junio de 2010 pertenecientes a la Fundación Salvadoreña para la lucha contra el SIDA "María Lorena" (CONTRASIDA) del municipio de San Salvador. (Bachelor thesis). Universidad de El Salvador.

Averous, P., Charbonnier, E., Lagouanelle-Simeoni, M., Prosperi, A., \& Dany, L. (2018). Illness perceptions and adherence in bipolar disorder: An exploratory study. Comprehensive Psychiatry, 80, 109-115. https://doi.org/10.1016/j. comppsych.2017.10.003

Bahamón, M., Vianchá, M., \& Tobos, A. (2014). Prácticas y conductas sexuales de riesgo en jóvenes: una perspectiva de género. Psicología desde el Caribe, 31(2), 327-353. http://www.scielo.org.co/scielo.php?script=sci_arttext\&pi$d=S 0123-417 X 2014000200008 \& \mid n g=e n \& \ln g=e s$

Bellack, J. P., \& Fleming, J. W. (1996). The use of projective techniques in pediatric nursing research from 1984 to 1993. Journal of pediatric nursing, 11(1), 10-28. https://doi.org/10.1016/S0882-5963(96)80034-0

Boon-Yasidhi, V., Kottapat, U., Durier, Y., Plipat, N., Phongsamart, W., Chokephaibulkit, K., \& Vanprapar, N. (2005). Diagnosis Disclosure in HIV-Infected Thai Children. Journal of the Medical Association of Thailand 88(8), 100-05. https:// www.ncbi.nlm.nih.gov/pubmed/16858851

Bustos, V., \& Russo, A. (2018). Salud mental como efecto del desarrollo psicoafectivo en la infancia. Psicogente, 21(39), 183-202 http://doi.org/10.17081/ psico.21.39.2830

Committee on Pediatric AIDS (1999). Disclosure of illness status to children and adolescents with HIV infection. American Academy of Pediatrics, 103(1), 164-166. https://doi.org/10.1542/peds.103.1.164

Corman, L. (1967). El test del Dibujo de la Familia en la Práctica Médico-Pedagógica. Buenos Aires: KAPELUSZ.

Croyle, R. T., \& Williams, K. D. (1991). Reactions to medical diagnosis: The role of illness stereotypes. Basic and Applied Social Psychology, 12(2), 227-241. https:// doi.org/10.1207/s15324834basp1202_7

Cummings, E. M., \& Davies, P. T. (2002). Effects of marital conflict on children: Recent advances and emerging these in process-oriented research. Journal of Child Psychology and Psychiatry, 43(1) https://doi.org/10.1111/1469-7610.00003

Ehrenzweig, Y. (2005). Modelos de cognición social y adherencia terapéutica en pacientes con cáncer. En L. Flores, M.M. Botero y B. Moreno-Jiménez (Eds.), 
Psicología de la salud: temas actuales de investigación en Latinoamérica (pp. 133-152). Barranquilla, Colombia: UNINORTE.

Fleming, J., \& Bellack, J. (1996). The use of projective techniques in pediatric nursing research from 1984 to 1993. Journal of Pediatric Nursing, (11), 10-28. https://doi. org/10.1016/S0882-5963(96)80034-0

Frostholm, L., Fink, P., Christensen, K., Toft, T., Oernboel, E., Olesen, F., \& Weinman, J. (2005). The patients' illness perceptions and the use of primary health care. Psychosomatic Medicine, 67(6), 997-1005. https://doi.org/10.1097/01. psy.0000189164.85653.bc

Guevara-Sotelo, Y., \& Hoyos-Hernández, P. A. (2018). Vivir con VIH: experiencias de estigma sentido en personas con VIH. Psicogente, 21(39), 127-139. https://doi. org/10.17081/psico.21.39.2827

Havens J.F., Mellins C.A. \& Hunter J. (2002). Psychiatric aspects of HIV/AIDS in childhood and adolescence. In: M. Rutter, E. Taylor (Eds), Child and adolescent psychiatry: Modern approaches. pp.828-841. 4th ed. Maiden, MA: Blackwell.

Hernández Sampieri, R., Fernández Collado, C., \& Baptista Lucio, M. (2010). Metodología de la investigación. Quinta edición. México D.F: McGraw-Hill.

Instituto Colombiano de Bienestar Familiar, Save the Children, Unicef, \& Universidad del Norte. (2006). Calidad de vida, apoyo social y utilización de servicios de salud y educación en niños, niñas y adolescentes y sus acudientes afectados con VIH/ SIDA en cinco ciudades-regiones colombianas: Cali y Buenaventura y Barranquilla, Santa Marta y Cartagena. Atlántico. Barranquilla: Universidad del Norte: Dirección de Investigaciones y Proyectos (DIP) Universidad del Norte.

Instituto Nacional de Salud (2017). Informe del comportamiento en la notificación de VIH-sida hasta el periodo epidemiológico del año 2017. Bogotá: Instituto Nacional de Salud. https://www.ins.gov.co/buscador-eventos/BoletinEpidemiologico/2018\%20Bolet\%C3\%ADn\%20epidemiol\%C3\%B3gico\%20semana\%2047. pdf

Instone, S. L. (2000). Perceptions of children with HIV infection when not told for so long: Implications for diagnosis disclosure. Journal of Pediatric Health Care, (14), 235-243. https://doi.org/10.1067/mph.2000.107338

Kouyoumdjian, F., Meyers, T., \& Mtshizana, S. (2005). Barriers to disclosure to children with HIV. Journal of Tropical Pediatrics 51(5), 285-287. https://doi.org/10.1093/ tropej/fmi014

Kwok, S., \& Shek, D. (2009). Social problem solving, family functioning, and suicidal ideation among Chinese adolescents in Hong Kong. Adolescence, 44(174), 391-406. https://www.ncbi.nlm.nih.gov/pubmed/19764274

Ledlie, S. (1999). Diagnosis Disclosure by Family Caregivers to Children who have Perinatally Acquired HIV Disease: Whenthe Time Comes. Nursing Research, 48(3), 141-9. https://www.ncbi.nlm.nih.gov/pubmed/10337845

Lee C, Johann-Liang R.(1999) Disclosure of the diagnosis of HIV/AIDS to children born of HIV-infected mothers. AIDS Patient Care STDS. 13(1) pp.41-45.

Lester, P., Chesney, M., Cooke, M., Weiss, R., Whaley, P., Pérez, B., \& Wara, D. (2002). Factors associated with diagnostic disclosure and emotional distress in HIV-infected children. Journal of Acquired Inmune Deficiency Syndromes, 31(3), 309-17. https://doi.org/10.1097/00126334-200211010-00006

Lipson, M. (1993). What do you say a child with AIDS. The Hastings Center Report, 23(2), 50. https://doi.org/10.2307/3562811 
Lodo-Platone, M. (2007). El test del dibujo de la familia: cuantificación y análisis de la estructura y dinámica familiar a través de la representación gráfica en escolares del área metropolitana. Caracas: Venezuela: Fondo Editorial Humanidades.

Mass, L. (2013). Familia y maltrato infantil: Una revisión teórica en torno a la clínica contemporánea. Revista CES, 4(2), 35-43. https://revistascientificas.cuc.edu.co/ culturaeducacionysociedad/article/view/982

Montoya, D., Pinilla, V., \& Dussán, C. (2018). Caracterización del autoconcepto en una muestra de estudiantes universitarios de algunos programas de pregrado de la ciudad de Manizales. Psicogente, 21(39), 162-182. http://doi.org/10.17081/ psico.21.39.2829

Nehring, W., Lashley, F., \& Malm, K. (2000). Disclosing the diagnosis of pediatric HIV infection: mother's views. Journal of the Society of Pediatric Nurses, 5(1), 5-14. https://doi.org/10.1111/j.1744-6155.2000.tb00081.x

Nettleton, S. (2002). Sociología de la salud y de la enfermedad. Atenas: Tipothito-Dardanos.

Oberdorfer, P., Puthanakit, T., Louthrenoo, O., Charnsil, C., Sirisanthana, T., \& Sirisanthana, V. (2006).Disclosure of HIV/AIDS diagnosis to HIV-infected children in Thailand. Journal of Pediatrics and Child Health, 42(5), 283-8. https://doi. org/10.1111/j.1440-1754.2006.00855.x

ONUSIDA (2016). HIV and AIDS Estimates. Bogotá: ONUSIDA.

Pérez Quiroz, A., Uribe Alvarado, I., Vianchá, M., Bahamón Muñetón, M., Verdugo Lucero, J., \& Ochoa Alcaráz, S. (2013). Estilos parentales como predictores de ideación suicida en estudiantes adolescentes. Psicología desde el caribe, 30(3), 551-568. http://www.scielo.org.co/scielo.php?script=sci_arttext\&pi$d=S 0123-417 X 2013000300006$

Petrie, K. J., Jago, L. A., \& Devchic, D. C. (2007). The Role of Illness Perceptions in Patients with Medical Conditions. Curr Opin Psychiatry, 20(2), 163-167. https:// doi.org/10.1097/YCO.0b013e328014a871

Portuondo, J. (2007). La Figura Humana Test Proyectivo de Karen Machover. Madrid: Biblioteca Nueva.

Sánchez de Gallardo, M., \& Pírela de Faría, L. (2012). Estudio psicométrico de la prueba figura humana. Telos, 14(2), 210-222.

Tasker, M. (1992). How I Can Tell You? Secrecy and Disclosure with Children When a Family Member Has AIDS. Maryland: Association for the Care of Children's Health.

Taylor, S. (2007). Psicología de la Salud. México, D.F: McGraw-Hill.

Trejos Herrera, A. M., Alarcón Vásquez, Y., Reyes Ruiz, L., \& Bahamón, M. J. (2017). ¿Por qué no se revela el diagnóstico a los niños y niñas seropositivos para $\mathrm{VIH} /$ SIDA? Factores asociados a la revelación y no revelación en Cuidadores y Profesionales de la Salud. En A. M. Trejos Herrera, L. Reyes Ruiz, Y. Alarcón Vásquez, M. J. Bahamón, O. González Gutiérrez, G. G. Gaviria García, \& A. J. Acosta Hoyos. El derecho de los niños y las niñas a conocer su diagnóstico de VIH/SIDA: la revelación del diagnóstico a través del modelo clínico "DIRÉ" (p.53). Barranquilla, Colombia: Ediciones Universidad Simón Bolívar. http://bonga.unisimon.edu.co/ handle/123456789/1139

Trejos Herrera, A. M., Alarcón Vásquez, Y., Bahamón, M. J., \& Reyes Ruiz, L. (2017). ¿Qué saben los niños y niñas seropositivos para VIH/SIDA antes de la revelación del diagnóstico? En A. Trejos Herrera, Y. Alarcón Vásquez, M. J. Bahamón, L. Reyes Ruíz, O. González Gutiérrez, A. J. Acosta Hoyos, \& G. G. Gaviria García, El derecho de los niños y las niñas a conocer su diagnóstico de VIH/SIDA: la revelación del diagnóstico a través del modelo clínico "DIRÉ" (p.68). Barranquilla, Colombia: 
Ediciones Universidad Simón Bolívar. URL: http://bonga.unisimon.edu.co/ handle/123

Trejos Herrera, A. M., Bahamón, M. J., \& Gaviria García, G. G. (2017). Beneficios de la revelación del diagnóstico de VIH/SIDA para las familias afectadas. En M. J. Bahamón, Y. Alarcón Vásquez, L. Reyes Ruíz, O. González Gutiérrez, A. J. Acosta Hoyos, G. G. Gaviria García, \& A. M. Trejos Herrera, El derecho de los niños y las niñas a conocer su diagnóstico de VIH/SIDA: la revelación del diagnóstico a través del modelo clínico "DIRÉ" (p.84). Barranquilla, Colombia: Ediciones Universidad Simón Bolívar. http://bonga.unisimon.edu.co/handle/123456789/1139

Trejos, A. M., Mosquera, M., \& Tuesca, R. (2009). Niñez afectada con VIH/SIDA: Calidad de vida, funcionalidad familiar y apoyo social en cinco ciudades colombianas. Salud, UniNorte, 25(1), 17-32. http://www.redalyc.org/articulo. oa? id $=81711840003$

Trejos, A. M., Reyes, L., Bahamón, M. J., Alarcón, Y., \& Gaviria, G. (2015). Efectos en la adherencia al tratamiento y en el ajuste psicológico luego de la revelación del diagnóstico de VIH/SIDA con el modelo clínico "DIRÉ" en niños y jóvenes colombianos menores de 17 años. Rev. Chilena de Infectología, 32(4), 408-415. https:// dx.doi.org/10.4067/S0716-10182015000500007

Trejos, A. M., Reyes, L., Bahamón, M., \& Alarcón, Y. (2014). Patterns of HIV status disclosure and nondisclosure to children under 17 years in health professionals and caregivers participants in "DIRÉ" clinical model in Barranquilla-Colombia. Pensee Journal, 76(12), 148-160. http://www.penseejournal.com/auto/index. $\mathrm{php} / \mathrm{pdf} /$ stream/XbLOIxX/1417554941

UNAIDS (2017). Ending AIDS: Progress towards the 90-90-90 Targets. Bogotá: UNAIDS. http://www.unaids.org/en/resources/documents/2017/20170720_ Global_AIDS_update_2017

Uribe Alvarado, I., Reyes Ruíz, L., Trejos Herrera, A. M., Bahamón Muñetón, M. J., \& Alarcón Vásquez, Y. (2017). Percepción de autoeficacia, asertividad sexual y uso del condón en jóvenes colombianos. Acta Colombiana de Psicología, 20(1), 212-220. http://www.dx.doi.org/10.14718/ACP.2017.20.1.10

Varas, N., Serrano, I., \& Toro, J. (2004). Estigma y diferencia social: VIH/SIDA en Puerto Rico. San Juan, PR: Huracanes.

Varela, M. T., Salazar, I. C., \& Correa, D. (2008). Adherencia al tratamiento en la infección por VIH/SIDA. Consideraciones teóricas y metodológicas para su abordaje. Acta Colombiana de Psicología, 11(2), 101-14. http://www.scielo.org.co/scielo.php?script=sci_arttext\&pid=S0123-91552008000200010\&/ng=en\&tlng=.

Waugh,S. (2003).Parentalviews on disclosure of diagnosistotheirHIV-positivechildren. AIDS Care, 15(2), pp.169-76. https://doi.org/10.1080/0954012031000068317

Weinman, J., Olesen, F., Toft, T., Christensen, K., Oernboel, E., Fink, P., \& Frostholm, L. (2005). The uncertain consultation and patient satisfaction: The impact of patients' illness perceptions and a randomized controlled trial on the training of physicians' communication skills. Psychosomatic Medicine, 67(6), 897-905. https://doi.org/10.1097/01.psy.0000188403.94327.5b

\section{(cc) BY}




\section{ANEXO}

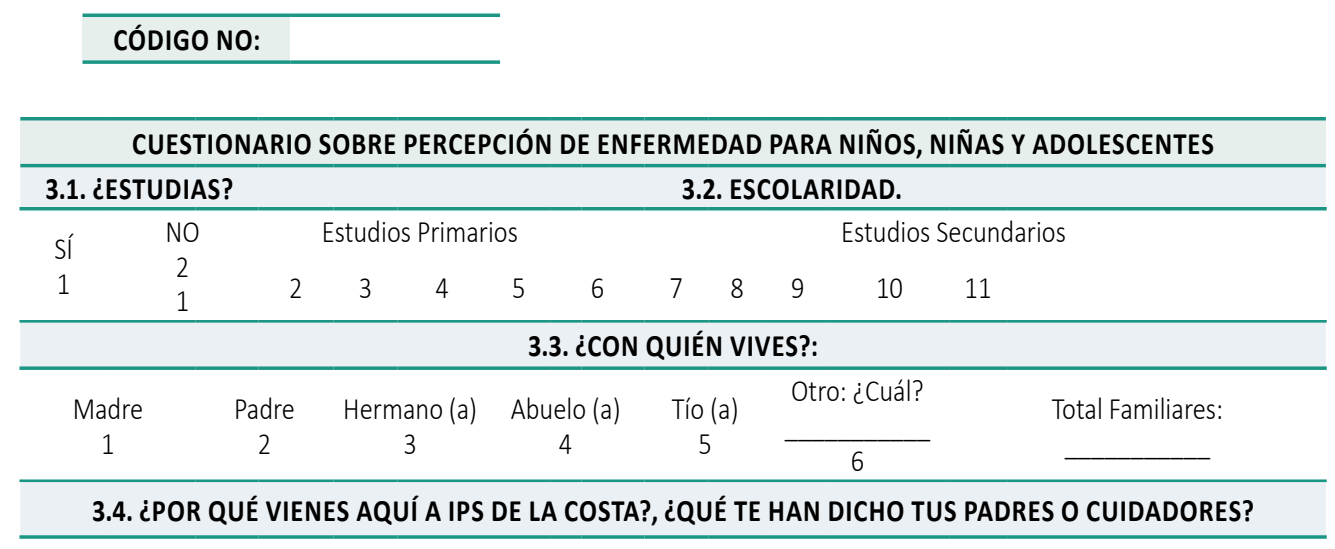

3.5. ¿CÓMO TE DIO ESA ENFERMEDAD Y/O SÍNTOMA?

3.6. PARA TI, ¿QUÉ ES LO PEOR DE ESTAR ENFERMO?

\section{7. ¿TOMAS ALGÚN MEDICAMENTO? (EN CASO QUE EL NIÑO O NIÑA RESPONDA POSITIVAMENTE PREGUNTE} NOMBRES DE LOS MEDICAMENTOS, DOSIS Y HORARIOS).

\section{8. ¿PARA QUÉ SON ESOS MEDICAMENTOS?:}

3.9. PARA TI, ¿QUÉ ES UNA ENFERMEDAD?:

\subsection{0. ¿QUÉ ENTIENDES ACERCA DE ESTAR ENFERMO?:}

3.11. ¿QUÉ ENTIENDES ACERCA DE ESTAR SANO O SALUDABLE?: 